

\title{
The Picard group of $M_{1,1}$
}

\author{
William Fulton and Martin Olsson
}

We compute the Picard group of the moduli stack of elliptic curves and its canonical compactification over general base schemes.

\section{Introduction}

Let $M_{1,1}$ denote the moduli stack (over $\mathbb{Z}$ ) classifying elliptic curves, and for a scheme $S$ let $M_{1,1, S}$ denote the fiber product $S \times \operatorname{Spec(\mathbb {Z})} M_{1,1}$. Mumford [1965] computed the Picard group $\operatorname{Pic}\left(M_{1,1, S}\right)$ when $S$ is the spectrum of a field of characteristic not 2 or 3 and found it to be cyclic of order 12. Our aim in this paper is to compute the Picard group Pic $\left(\mathcal{M}_{1,1, S}\right)$ for more general base schemes $S$, as well as to compute the Picard group Pic $\left(\bar{M}_{1,1, S}\right)$ for the standard compactification $\bar{M}_{1,1}$ of $M_{1,1}$.

Recall that on $M_{1,1}$ there is the Hodge bundle $\lambda$. For any morphism $t: T \rightarrow M_{1,1}$ corresponding to an elliptic curve $f: E \rightarrow T$, the pullback $t^{*} \lambda$ is the line bundle $f_{*} \Omega_{E / T}^{1}$. Equivalently, if $f: \mathscr{E} \rightarrow M_{1,1}$ denotes the universal elliptic curve, then $\lambda=f_{*} \Omega_{\mathscr{E} / M_{1,1}}^{1}$. This bundle extends canonically to $\bar{M}_{1,1}$. Namely, let $\bar{f}: \overline{\mathscr{E}} \rightarrow$ $\bar{M}_{1,1}$ denote the extension of $\mathscr{E}$ provided by the Tate curve and let $\omega_{\overline{\mathscr{E}} / \overline{\mathcal{M}}_{1,1}}$ denote the relative dualizing sheaf. Then the sheaf $\bar{f}_{*} \omega_{\overline{\mathscr{c}} / \bar{M}_{1,1}}$ is a line bundle on $\bar{M}_{1,1}$ extending $\lambda$. In what follows we will abuse notation and write also $\lambda$ for this line bundle on $\bar{M}_{1,1}$.

Recall the following basic facts and definitions about elliptic curves (see for example [Deligne 1975, §1]). If $\Lambda$ is a ring and $t: \operatorname{Spec}(\Lambda) \rightarrow M_{1,1}$ is a morphism corresponding to an elliptic curve $E / \Lambda$, then after replacing $\Lambda$ by an étale extension the family $E$ can be described by an equation

$$
y^{2}+a_{1} x y+a_{3} y=x^{3}+a_{2} x^{2}+a_{4} x+a_{6} .
$$

Define

$$
\begin{gathered}
b_{2}=a_{1}^{2}+4 a_{2}, \quad b_{4}=a_{1} a_{3}+2 a_{4}, \quad b_{6}=a_{3}^{2}+4 a_{6}, \\
b_{8}=-a_{1} a_{3} a_{4}-a_{4}^{2}+a_{1}^{2} a_{6}+a_{2} a_{3}^{2}+4 a_{2} a_{6},
\end{gathered}
$$

MSC2000: primary 14D22; secondary 14D05.

Keywords: moduli spaces, stacks, Picard group.

Olsson was partially supported by NSF grant DMS-0555827 and an Alfred P. Sloan fellowship. 
and the discriminant

$$
\Delta=-b_{2}^{2} b_{8}-8 b_{4}^{3}-27 b_{6}^{2}+9 b_{2} b_{4} b_{6} \in \Lambda^{*} .
$$

With these chosen coordinates a basis for $t^{*} \lambda$ is given by the invariant differential

$$
\pi=d x /\left(2 y+a_{1} x+a_{3}\right) .
$$

Any two choices of coordinates (1-1) differ by a transformation

$$
x^{\prime}=u^{2} x+r, \quad y^{\prime}=u^{3} y+s u^{2} x+t,
$$

where $u \in \Lambda^{*}$ and $r, s, t \in \Lambda$. One can compute that the invariant differential $\pi^{\prime}$ obtained from the coordinates $\left(x^{\prime}, y^{\prime}\right)$ is equal to $u^{-1} \pi$, and that the discrimant $\Delta^{\prime}$ in the coordinates $\left(x^{\prime}, y^{\prime}\right)$ is equal to $u^{12} \Delta$. In particular, the element $\Delta \pi^{\otimes 12} \in t^{*} \lambda^{\otimes 12}$ is independent of the choice of coordinates, and therefore defines a trivialization of $\lambda^{\otimes 12}$ over $M_{1,1}$.

Let $p: M_{1,1, S} \rightarrow \mathbb{A}_{S}^{1}$ be the map defined by the $j$-invariant

$$
j=\left(b_{2}^{2}-24 b_{4}\right)^{3} / \Delta .
$$

Theorem 1.1. Let $S$ be a scheme. Then the map

$$
\mathbb{Z} /(12) \times \operatorname{Pic}\left(\mathbb{A}_{S}^{1}\right) \rightarrow \operatorname{Pic}\left(M_{1,1, S}\right), \quad(i, \mathscr{L}) \mapsto \lambda^{\otimes i} \otimes p^{*} \mathscr{L}
$$

is an isomorphism if either of the following hold:

(i) $S$ is a $\mathbb{Z}[1 / 2]$-scheme.

(ii) $S$ is reduced.

Remark 1.2. As we observe in Remark 6.4 the theorem fails for nonreduced schemes in characteristic 2 .

Theorem 1.3. The map

$$
\mathbb{Z} \times \operatorname{Pic}(S) \rightarrow \operatorname{Pic}\left(\bar{M}_{1,1, S}\right), \quad(n, M) \mapsto \lambda^{n} \otimes_{\mathcal{O}_{S}} M
$$

is an isomorphism for any scheme $S$.

The paper is organized as follows. In Section 2 we give a proof of Theorem 1.1 in the case when 6 is invertible on the base. In this case the orders of the stabilizer groups of $\mu_{1,1, S}$ are invertible on $S$ (the stack $\mu_{1,1, S}$ is tame), which makes it easy to relate the invertible sheaves on $M_{1,1, S}$ and on its coarse moduli space (see Lemma 2.3). In Section 3 we then give a separate argument proving Theorem 1.1 which works when the base $S$ is normal. Using this case of a normal base, we then prove Theorem 1.1 when $S$ is reduced in Section 4. Once the case of reduced $S$ is established, the general statement of Theorem 1.1 reduces to studying the relationship between deformation theory of line bundles on $M_{1,1, S}$ and its coarse 
space. This essentially amounts to computing the coherent cohomology groups of $M_{1,1, S}$, which occupies Section 5. Finally in Section 6 we use similar techniques to prove Theorem 1.3.

Remark 1.4. By standard limit arguments it suffices to prove the above results in the case when $S$ is of finite type over an excellent Dedekind ring. In what follows we will therefore restrict ourselves to such schemes unless otherwise stated.

\section{When 6 is invertible on $S$}

Though the case when 6 is invertible follows from the more technical work in subsequent sections, we include here a proof in the case of a $\mathbb{Z}[1 / 6]$-scheme since it is much easier than the more general cases.

The following lemma is well-known (and does not require that 6 be invertible in $S$ ), but we include a proof for the convenience of the reader.

Lemma 2.1. For any locally noetherian scheme $S$, the map $\bar{\pi}: \bar{M}_{1,1, S} \rightarrow \mathbb{P}_{S}^{1}$ (resp. $\pi: M_{1,1, S} \rightarrow \mathbb{A}_{S}^{1}$ ) given by the $j$-invariant identifies $\mathbb{P}_{S}^{1}$ (resp. $\mathbb{A}_{S}^{1}$ ) with the coarse moduli space of $\bar{M}_{1,1, S}$ (resp. $\left.\mathcal{M}_{1,1, S}\right)$.

Proof. Since the square

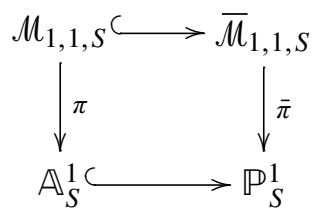

is cartesian, it suffices to show that $\bar{\pi}$ identifies $\mathbb{P}_{S}^{1}$ with the coarse moduli space of $\bar{M}_{1,1, S}$.

Let $\tilde{\bar{\pi}}: \bar{M}_{1,1, S} \rightarrow X$ be the coarse moduli space (which exists by [Keel and Mori 1997]). By the universal property of the coarse moduli space, there exists a unique morphism $f: X \rightarrow \mathbb{P}_{j}^{1}$ such that $f \circ \tilde{\bar{\pi}}=\bar{\pi}$. Since $\bar{\pi}$ is proper and quasifinite, the morphism $f$ is also proper and quasifinite and therefore $f$ is finite. Furthermore, by [Abramovich and Vistoli 2002, 2.2.1] we have $\tilde{\bar{\pi}}_{*} \mathcal{O}_{\bar{M}_{1,1, S}}=\mathcal{O}_{X}$. It therefore suffices to show that the map $\mathbb{O}_{\mathbb{P}_{j}^{1}} \rightarrow \bar{\pi}_{*} \widehat{O}_{\bar{M}_{1,1, S}}$ is an isomorphism. It suffices to verify this locally in the flat topology on $S$, so we may further assume that $S$ is the spectrum of a complete noetherian local ring $A$. In addition, since the morphism $\bar{\pi}$ is proper, the theorem on formal functions for stacks [Olsson 2005, §3] implies that it suffices to show the result over $\operatorname{Spec}\left(A / \mathfrak{m}_{A}^{n}\right)$ for all $n$. This reduces the proof to the case when $S$ is the spectrum of an artinian local ring $A$. Let $k$ be the residue field of $A$, and let $J \subset A$ be an ideal with $J$ annihilated by the maximal ideal of $A$ (so that $J$ is a $k$-vector space). Set $A_{0}:=A / J$. Pushing forward the 
exact sequence

$$
0 \rightarrow J \otimes \mathrm{O}_{\bar{M}_{1,1, k}} \rightarrow \mathrm{O}_{\bar{M}_{1,1, A}} \rightarrow \mathrm{O}_{\bar{M}_{1,1, A}} \rightarrow 0
$$

to $\mathbb{P}_{A}^{1}$ we obtain a commutative diagram

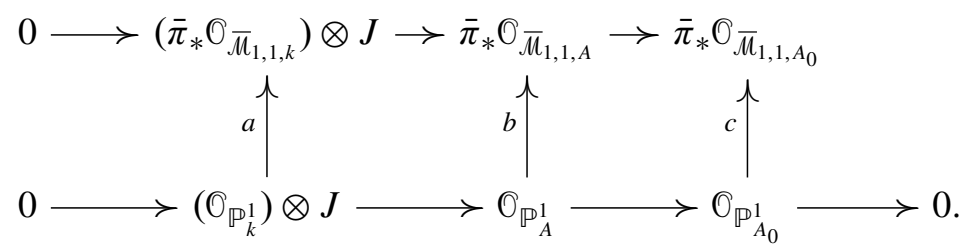

By induction and the case when $A$ is a field, we get that $a$ and $c$ are isomorphisms and therefore $b$ is an isomorphism also.

Assume for the rest of this section that $S$ is a $\mathbb{Z}[1 / 6]$-scheme.

Let $\tilde{s}_{4}: S \rightarrow M_{1,1, S}$ be the section corresponding to the elliptic curve with automorphism group $\mu_{4}\left(y^{2}=x^{3}+x\right.$ with $\left.\Delta=-64, j=1728\right)$ and $\tilde{s}_{6}: S \rightarrow$ $M_{1,1, S}$ the section corresponding to the elliptic curve with automorphism group $\mu_{6}$ $\left(y^{2}+y=x^{3}\right.$ with $\left.\Delta=-27, j=0\right)$. These sections define closed immersions $s_{4}: B \mu_{4, S} \hookrightarrow M_{1,1, S}$ and $s_{6}: B \mu_{6, S} \hookrightarrow M_{1,1, S}$. For any line bundle $\mathscr{L}$ on $M_{1,1, S}$ the pullback $s_{4}^{*} \mathscr{L}$ (resp. $s_{6}^{*} \mathscr{L}$ ) corresponds to a line bundle $M_{4}$ (resp. $M_{6}$ ) on $S$ with action of the group $\mu_{4}$ (resp. $\left.\mu_{6}\right)$. We thus get maps

$$
\rho_{4}: \mu_{4} \rightarrow \underline{\operatorname{Aut}}\left(M_{4}\right) \simeq \mathbb{G}_{m}, \quad \rho_{6}: \mu_{6} \rightarrow \underline{\operatorname{Aut}}\left(M_{6}\right) \simeq \mathbb{G}_{m}
$$

defining characters $\chi_{4} \in \mathbb{Z} /(4)$ and $\chi_{6} \in \mathbb{Z} /(6)$.

Lemma 2.2. The pair $\left(\chi_{4}, \chi_{6}\right)$ lies in $\mathbb{Z} /(12) \subset \mathbb{Z} /(4) \times \mathbb{Z} /(6)$.

Proof. The construction of the pair $\left(\chi_{4}, \chi_{6}\right)$ commutes with arbitrary base change on $S$, so it suffices to consider the case when $S$ is the spectrum of an algebraically closed field, $S=\operatorname{Spec}(k)$. We have to show that $\left.\rho_{4}\right|_{\mu_{2}}=\left.\rho_{6}\right|_{\mu_{2}}$. Write $k \llbracket t \rrbracket$ for the completion of the local ring of $\mathbb{A}_{j}^{1}$ at $j=1728$ and let $k \llbracket z \rrbracket$ be the completion of the local ring of $M_{1,1, S}$ at the point coresponding to the curve $y^{2}=x^{3}+x$. Then for suitable choices of the coordinates $t$ and $z$ the map $k \llbracket t \rrbracket \rightarrow k \llbracket z \rrbracket$ sends $t$ to $z^{2}$, and the action of $\mu_{4}$ is given by $\zeta * z=\zeta^{2} \cdot z$. Furthermore we can write $\left.\mathscr{L}\right|_{k \llbracket z \|}=k \llbracket z \rrbracket \cdot e$ for some basis $e$, such that $\rho_{4}$ acts by $\zeta * e=\zeta^{\chi_{4}} e$. From this we see that $\left.\rho_{4}\right|_{\mu_{2}}$ is equal to the character defined by the action of $\mu_{2}$ on the fiber of $\mathscr{L}$ at the generic point of $M_{1,1, S}$. Similarly, $\left.\rho_{6}\right|_{\mu_{2}}$ is equal to the action on the generic fiber.

We therefore obtain a map

$$
\operatorname{Pic}\left(M_{1,1, S}\right) \rightarrow \mathbb{Z} /(12), \quad \mathscr{L} \mapsto\left(\chi_{4}, \chi_{6}\right),
$$

and it follows from the construction that this map is a homomorphism. Let $K$ denote the kernel. 
Recall that a Deligne-Mumford stack $\mathscr{L}$ is called tame if for every algebraically closed field $\Omega$ and point $\bar{x}: \operatorname{Spec}(\Omega) \rightarrow \mathscr{X}$ the order of the automorphism group of $\bar{x}$ is relatively prime to the characteristic of $\Omega$.

Lemma 2.3. Let $\mathscr{X}$ be a tame Deligne-Mumford stack with coarse moduli space $\pi: \mathscr{X} \rightarrow X$. Let $\mathscr{L}$ be an invertible sheaf on $\mathscr{X}$ such that for every geometric point $\bar{x} \rightarrow \mathscr{L}$ the action of the stabilizer group $G_{\bar{x}}$ on $\mathscr{L}(\bar{x})$ is trivial. Then $\pi_{*} \mathscr{L}$ is an invertible sheaf on $X$ and $\pi^{*} \pi_{*} \mathscr{L} \rightarrow \mathscr{L}$ is an isomorphism.

Proof. It suffices to prove the lemma after passing to the strict henselization of $X$ at a geometric point $\bar{x}$. Let $A=\mathcal{O}_{X, \bar{x}}$ and $B=\mathcal{O}_{\mathscr{X}, \bar{x}}$. Then, as explained in [Olsson 2006, 2.12], if $\Gamma$ denotes the stabilizer group of $\bar{x}$ then there is a natural action of $\Gamma$ on $B$ such that $\mathscr{X}=[\operatorname{Spec}(B) / \Gamma]$. Let $M$ be the free $B$-module with $\Gamma$-action of rank 1 defining $\mathscr{L}$. Since $\Gamma$ has order invertible in $k(\bar{x})$ (since $\mathscr{X}$ is tame), the representation category of $\Gamma$ is semisimple. By our assumptions, the reduction $M \otimes k(\bar{x})$ is generated by an invariant element, and choosing a lifting to an invariant element of $M$ we see that we can write $M=B \cdot e$ where $\Gamma$ acts trivially on $e$. Then $\pi_{*} \mathscr{L}$ is just $A \cdot e$ and the lemma is immediate.

Corollary 2.4. The homomorphism $\pi^{*}: \operatorname{Pic}\left(\mathbb{A}_{S}^{1}\right) \rightarrow K$ is an isomorphism.

Proof. We show that if $\mathscr{L}$ is a line bundle with $\left(\chi_{4}, \chi_{6}\right)=(0,0)$, then $\pi_{*} \mathscr{L}$ is an invertible sheaf on $\mathbb{A}_{S}^{1}$ and $\pi^{*} \pi_{*} \mathscr{L} \rightarrow \mathscr{L}$ is an isomorphism. By Lemma 2.3 it suffices to show that for any geometric point $\bar{x} \rightarrow M_{1,1, S}$, the action of the stabilizer group of $\bar{x}$ on $\mathscr{L}(\bar{x})$ is trivial. For this we may assume that $S$ is the spectrum of an algebraically closed field. By our assumptions, the actions $\rho_{4}$ and $\rho_{6}$ are trivial. By the argument used in the proof of Lemma 2.2, this implies that the action of the generic stabilizer is also trivial. From this it follows that the action is trivial at every point since over $\mathbb{A}^{1}-\{0,1728\}$ the stack $\mu_{1,1, S}$ is a $\mu_{2}$-gerbe.

Lemma 2.5. The image of $\lambda$ in $\mathbb{Z} /(12)$ is a generator. In particular (2-3) is surjective.

Proof. It suffices to consider the case when $S$ is the spectrum of a field, in which case the above shows that $\operatorname{Pic}\left(M_{1,1, S}\right)$ injects into $\mathbb{Z} /(12)$. We can in fact compute directly the image of $\lambda$ in $\mathbb{Z} /(4) \times \mathbb{Z} /(6)$. The image in $\mathbb{Z} /(4)$ corresponds to the representation of $\mu_{4}$ given by the action on the invariant differential $d x / 2 y$ of the curve $y^{2}=x^{3}+x$. An element $\zeta \in \mu_{4}$ acts by $(x, y) \mapsto\left(\zeta^{2} x, \zeta y\right)$, and therefore the action on $d x / 2 y$ is equal to multiplication by $\zeta$. Therefore the image of $\lambda$ in $\mathbb{Z} /(4)$ is equal to 1 .

Similarly, the image of $\lambda$ in $\mathbb{Z} /(6)$ corresponds to the character given by the invariant differential $d x /(2 y+1)$ of the curve $y^{2}+y=x^{3}$. Write $\mu_{6}=\mu_{2} \times \mu_{3}$. Then $(-1,1)$ acts by $(x, y) \mapsto(x,-y-1)$ and $(1, \zeta)$ acts by $(x, y) \mapsto(\zeta x, y)$. Therefore $(-1,1)$ acts on the invariant differential by multiplication by -1 and 
$(1, \zeta)$ acts by multiplication by $\zeta$. It follows that $\lambda$ maps to 1 in $\mathbb{Z} /(6)$ which implies that $\lambda$ is a generator in $\mathbb{Z} /(12)$.

Corollary 2.6. The map $\lambda \times \pi^{*}:(\mathbb{Z} / 12) \times \operatorname{Pic}\left(\mathbb{A}_{S}^{1}\right) \rightarrow \operatorname{Pic}\left(M_{1,1, S}\right)$ is an isomorphism.

\section{The case of a normal affine scheme $S$}

Write $S=\operatorname{Spec}(\Lambda)$ with $\Lambda$ a normal ring. Let $U$ be the scheme

$$
U:=\operatorname{Spec}\left(\Lambda\left[a_{1}, a_{2}, a_{3}, a_{4}, a_{6}\right][1 / \Delta]\right),
$$

where $\Delta$ is as in (1-3). Equation (1-1) defines a family of elliptic curves $E \rightarrow U$. Let $G$ denote the group scheme with underlying scheme $\operatorname{Spec}\left(\Lambda\left[u^{ \pm}, r, s, t\right]\right)$ with group law defined by

$$
\left(u^{\prime}, r^{\prime}, s^{\prime}, t^{\prime}\right) \cdot(u, r, s, t)=\left(u u^{\prime}, u^{2} r^{\prime}+r, u s^{\prime}+s, u^{3} t^{\prime}+u^{2} r^{\prime} s+t\right) .
$$

The group $G$ acts on $E$ over the action on $U$ via the formulas [Deligne 1975, 1.6], and $\mathcal{M}_{1,1, S}$ is isomorphic to the stack theoretic quotient $[U / G]$.

Proposition 3.1. The pullback map

$$
\operatorname{Pic}(S) \rightarrow \operatorname{Pic}(U)
$$

is an isomorphism.

Proof. The key point is the following result of Ischebeck [1979, §4].

Lemma 3.2. Let $\Delta \in \mathbb{Z}\left[t_{1}, \ldots, t_{n}\right]$ be a polynomial satisfying

(i) The greatest common divisor of the coefficients of its nonconstant monomials is 1 .

(ii) For any field $k$ the image of $\Delta$ in $k\left[t_{1}, \ldots, t_{n}\right]$ is irreducible.

Then for any noetherian normal ring $\Lambda$, the pullback homomorphism

$$
\operatorname{Pic}(\Lambda) \rightarrow \operatorname{Pic}\left(\Lambda\left[t_{1}, \ldots, t_{n}\right][1 / \Delta]\right)
$$

is an isomorphism.

Proof. The assumptions are used as follows:

(1) Assumption (i) implies that the map $\operatorname{Spec}\left(\mathbb{Z}\left[t_{1}, \ldots, t_{n}\right][1 / \Delta]\right) \rightarrow \operatorname{Spec}(\mathbb{Z})$ is surjective and hence faithfully flat. It follows that the map

$$
\operatorname{Spec}\left(\Lambda\left[t_{1}, \ldots, t_{n}\right][1 / \Delta]\right) \rightarrow \operatorname{Spec}(\Lambda)
$$

is also faithfully flat. 
(2) By the preceding observation, the divisor $V(\Delta) \subset \operatorname{Spec}\left(\Lambda\left[t_{1}, \ldots, t_{n}\right]\right)$ does not contain any fibers, and by (ii) its generic fiber is nonempty and irreducible. From this it follows that $V(\Delta)$ is irreducible.

It follows that there is an exact sequence of Weil divisor class groups [Fulton 1998, $1.8]$

$$
\mathbb{Z}[V(\Delta)] \stackrel{0}{\rightarrow} \mathrm{Cl}\left(\Lambda\left[t_{1}, \ldots, t_{n}\right]\right) \rightarrow \mathrm{Cl}\left(\Lambda\left[t_{1}, \ldots, t_{n}\right][1 / \Delta]\right) \longrightarrow 0 .
$$

We conclude that

$$
\mathrm{Cl}(\Lambda) \simeq \mathrm{Cl}\left(\Lambda\left[t_{1}, \ldots, t_{n}\right]\right) \simeq \mathrm{Cl}\left(\Lambda\left[t_{1}, \ldots, t_{n}\right][1 / \Delta]\right) .
$$

The normality of $\Lambda$ implies that the natural maps from the Picard groups to the Weil divisor class groups are injective. Therefore, it suffices to show that if $D \in$ $\mathrm{Cl}(\Lambda)$ is a Weil divisor whose image in $\operatorname{Cl}\left(\Lambda\left[t_{1}, \ldots, t_{n}\right][1 / \Delta]\right)$ is in the image of $\operatorname{Pic}\left(\Lambda\left[t_{1}, \ldots, t_{n}\right][1 / \Delta]\right)$, then $D$ is obtained from a line bundle on $\operatorname{Spec}(\Lambda)$. This follows from the observation that $\Lambda \rightarrow \Lambda\left[t_{1}, \ldots, t_{n}\right][1 / \Delta]$ is faithfully flat [Ischebeck 1979, §4, Satz 6].

We apply the lemma to $\Delta \in \mathbb{Z}\left[a_{1}, \ldots, a_{6}\right]$; (i) is immediate and (ii) follows from the calculations in [Deligne 1975, §3, §4] (Note that though these sections concern characteristics prime to 6 , the same calculations give the irreducibility of $\Delta$ over arbitrary fields.)

The isomorphism $\mu_{1,1, S} \simeq[U / G]$ defines a morphism $\sigma: M_{1,1, S} \rightarrow B G$. For a character $\chi: G \rightarrow \mathbb{G}_{m}$ defining a line bundle on $B G$, let $L_{\chi}$ be the line bundle on $\mu_{1,1, S}$ obtained by pullback along $\sigma$.

Lemma 3.3. Let $\mathscr{L}$ be a line bundle on $M_{1,1, S}$ such that the pullback $L$ of $\mathscr{L}$ to $U$ is trivial. Then $\mathscr{L} \simeq L_{\chi}$ for some character $\chi: G \rightarrow \mathbb{G}_{m}$.

Proof. Fix a basis $e \in L$.

Let $\mathscr{F}$ be the sheaf on the category of affine $S$-schemes (with the étale topology) which to any morphism of affine schemes $S^{\prime} \rightarrow S$ associates $\Gamma\left(U_{S^{\prime}}, O_{U_{S^{\prime}}}^{*}\right)$. There is an inclusion of sheaves $\mathbb{G}_{m} \subset \mathscr{F}$ given by the inclusions $\Gamma\left(S^{\prime}, \mathbb{O}_{S^{\prime}}^{*}\right) \subset \Gamma\left(U_{S^{\prime}}, O_{U_{S^{\prime}}}^{*}\right)$. For any $S^{\prime} \rightarrow S$ and $g \in G\left(S^{\prime}\right)$, we get an element $u_{g} \in \mathscr{F}\left(S^{\prime}\right)$ defined by the condition that $g(e)=u_{g} \cdot e \in L$. This defines a map of sheaves (not necessarily a homomorphism)

$$
f: G \rightarrow \mathscr{F} \text {. }
$$

To prove the lemma it suffices to show that $f$ has image contained in $\mathbb{G}_{m} \subset \mathscr{F}_{\text {(note }}$ that it is clear that if this holds then the map $G \rightarrow \mathbb{G}_{m}$ is a homomorphism).

Since $G$ is an affine scheme, the map $f$ is determined by a section $u_{0} \in \mathscr{F}(G)$. Since $G$ is normal and connected, this section $u_{0} \in \Gamma\left(U_{G}, O_{U_{G}}^{*}\right)$ can be written uniquely as $\beta \Delta^{m}$, where $\beta \in \Gamma\left(G, O_{G}^{*}\right)$ and $m \in \mathbb{Z}$. We need to show that $m=0$. 
For this, note that the image of $u_{0}$ under the map $\mathscr{F}(G) \rightarrow \mathscr{F}(S)$ defined by the identity section $e: S \rightarrow G$ is equal to 1 . It follows that $e^{*}(\beta) \cdot \Delta^{m}$ is equal to 1 in $\Gamma\left(U, O_{U}^{*}\right)$ which implies that $m=0$.

Lemma 3.4. Any homomorphism $G \rightarrow \mathbb{G}_{m}$ factors through the projection

$$
\chi_{0}: G \rightarrow \mathbb{G}_{m}, \quad(u, r, s, t) \mapsto u .
$$

Proof. There are three injective homomorphisms

$$
j_{r}, j_{s}, j_{t}: \mathbb{G}_{a} \hookrightarrow G
$$

sending $x \in \mathbb{G}_{a}$ to $(1, x, 0,0),(1,0, x, 0)$, and $(1,0,0, x)$ respectively. The formula

$$
(1, r, 0,0)(1,0, s, 0)(1,0,0, t-r s)=(1, r, s, t)
$$

shows that the subgroup of $G$ generated by the images of these three inclusions is equal to the kernel of $\chi_{0}$. Since any homomorphism $\mathbb{G}_{a} \rightarrow \mathbb{G}_{m}$ is trivial, it follows that any homomorphism $G \rightarrow \mathbb{G}_{m}$ has kernel containing $\operatorname{Ker}\left(\chi_{0}\right)$.

Lemma 3.5. Let $n, m \in \mathbb{Z}$ be integers. Then $L_{\chi_{0}^{n}} \simeq L_{\chi_{0}^{m}}$ if and only if $n \equiv m$ $(\bmod 12)$.

Proof. Observe that

$$
L_{\chi_{0}^{n}} \otimes L_{\chi_{0}^{m}}^{-1} \simeq L_{\chi_{0}^{n-m}}
$$

so it suffices to show that $L_{\chi_{0}^{n}} \simeq \mathcal{O}_{\mathcal{M}_{1,1, S}}$ if and only if $12 \mid n$.

Choose a basis $e \in L_{\chi_{0}^{n}}(U)$ such that $G$ acts on $e$ through $\chi_{0}^{n}$. Then an isomorphism $0_{\mu_{1,1, S}} \simeq L_{\chi_{0}^{n}}$ is given by a function $\epsilon \in \Gamma\left(U, \mathscr{O}_{U}^{*}\right)$ such that the action of $G$ on $\epsilon^{-1} \cdot e$ is trivial. Equivalently we want a global section $\epsilon \in \Gamma\left(U, O_{U}^{*}\right)$ such that $G$ acts on $\epsilon$ through $\chi_{0}^{n}$. Since

$$
\Gamma\left(U, O_{U}^{*}\right) \simeq \Lambda^{*} \cdot \Delta^{\mathbb{Z}},
$$

and $G$ acts on $\Delta$ through $\chi_{0}^{12}$, such a unit $\epsilon$ exists if and only if $12 \mid n$.

Let $l: L_{\chi_{0}^{12}} \simeq \mathcal{O}_{\mu_{1,1, S}}$ be the trivialization defined by $\Delta$. This trivialization enables us to speak of $L_{\chi_{0}^{n}}$ for any class $n \in \mathbb{Z} /(12)$.

By Proposition 3.1, if $\mathscr{L}$ is a line bundle on $M_{1,1, S}$, then the pullback of $\mathscr{L}$ to $U$ is isomorphic to the pullback of a line bundle $M$ on $S$. Trivializing this line bundle $M$ locally on $S$, we see that locally on $S$ we have an isomorphism $\mathscr{L} \simeq L_{\chi_{0}^{n}}$, for a unique class $n \in \mathbb{Z} /(12)$. From the uniqueness it follows that to any connected $S$ and line bundle $\mathscr{L}$ on $M_{1,1, S}$, there exists a unique class $n \in \mathbb{Z} /(12)$ and line bundle $M$ on $S$ such that

$$
\mathscr{L} \simeq M \otimes L_{\chi_{0}^{n}} .
$$

Moreover, such a line bundle $\mathscr{L}$ is trivial if and only if $M$ is trivial and $n$ is the zero element of $\mathbb{Z} /(12)$. 
The line bundle $\lambda$ is trivialized over $U$ by the invariant differential $\pi$ defined in (1-4), and as mentioned in the introduction the action of $(u, r, s, t) \in G$ on $\pi$ is through the character $G \rightarrow \mathbb{G}_{m}$ sending $(u, r, s, t)$ to $u^{-1}$. Therefore

$$
\lambda \simeq L_{\chi_{0}^{-1}} .
$$

This completes the proof of Theorem 1.1 in the case when $S$ is affine and normal.

A very similar argument can be used to prove Theorem 1.3 in the case when the base scheme $S$ is affine and normal. Let $c_{4}=b_{2}^{2}-24 b_{4}$. Then one can show (see for example [Silverman 2009, III.1.4]) that (1-1) is nodal precisely when $\Delta=0$ and $c_{4} \neq 0$. Let $\widetilde{U}$ denote

$$
\operatorname{Spec}\left(\Lambda\left[a_{1}, a_{2}, a_{3}, a_{4}, a_{6}\right]\right)-V\left(\Delta, c_{4}\right) .
$$

Again, the group scheme $G$ acts on $\widetilde{U}$ and $\bar{M}_{1,1, S} \simeq[\widetilde{U} / G]$.

Lemma 3.6. (i) The map

$$
\operatorname{Pic}(\Lambda) \rightarrow \operatorname{Pic}(\widetilde{U})
$$

is an isomorphism.

(ii) The map $\Lambda^{*} \rightarrow \Gamma\left(\widetilde{U}, O_{\widetilde{U}}^{*}\right)$ is an isomorphism.

Proof. Statement (ii) is immediate. Statement (i) follows from a very similar argument to the proof of Proposition 3.1. The only new ingredient is that the polynomial $c_{4}$ is not irreducible over fields of characteristics 2 and 3 but it is a power of an irreducible polynomial (in characteristic 2 it is equal to $a_{1}^{4}$ and in characteristic 3 it is equal to $\left(a_{1}+a_{2}\right)^{2}$.

Using this, one sees as before that the map

$$
\operatorname{Pic}(\Lambda) \times \operatorname{Pic}(B G) \rightarrow \operatorname{Pic}\left(\bar{M}_{1,1, S}\right)
$$

is an isomorphism with the character Equation (3-9) mapping to $\lambda^{-1}$.

\section{The case when $S$ is reduced}

4.1. If $S$ is an arbitrary scheme, and $\mathscr{L}$ a line bundle on $\mathcal{M}_{1,1, S}$, then there is a unique function $s \mapsto l(s) \in \mathbb{Z} /(12)$ which associates to a point $s$ the unique power $l(s)$ of $\lambda$ such that $\mathscr{L}_{s} \otimes \lambda^{-l(s)}$ on $M_{1,1, k(s)}$ descends to $\mathbb{A}_{k(s)}^{1}$ (and hence is trivial).

Lemma 4.2. The function $s \mapsto l(s)$ is a locally constant function on $S$.

Proof. The assertion is local on $S$ so we may assume that $S$ is affine. Furthermore, the assertion can be verified on each irreducible component so we may assume that $S$ is integral. Finally, if $\widetilde{S} \rightarrow S$ is the normalization then it suffices to verify the assertion for $\widetilde{S}$. In this case the result follows from Section 3 . 
4.3. In particular, if $S$ is connected we obtain a homomorphism

$$
\operatorname{Pic}\left(M_{1,1, S}\right) \rightarrow \mathbb{Z} /(12)
$$

sending $\lambda$ to 1 . Thus, in general, to prove Theorem 1.1 we need to show that the kernel of (4-1) is isomorphic to $\operatorname{Pic}\left(\mathbb{A}_{S}^{1}\right)$.

4.4. To complete the proof of Theorem 1.1 in the case when $S$ is reduced, we make some general observations about the relationship between line bundles on a stack and line bundles on the coarse moduli space (Recall that by Lemma 2.1, the $j$-invariant identifies $\mathbb{A}_{S}^{1}$ with the coarse moduli space of $\mu_{1,1, S}$.)

Let $S$ be a noetherian scheme and $\mathscr{X} \rightarrow S$ a Deligne-Mumford stack over $S$. Let $\pi: \mathscr{X} \rightarrow X$ be the coarse moduli space, and assume that the formation of the coarse space $X$ commutes with arbitrary base change on $S$ and that $X$ is reduced (we just saw that this holds for $M_{1,1}$ over a reduced scheme). For a field valued point $x: \operatorname{Spec}(k) \rightarrow S$, let $\pi_{x}: \mathscr{X}_{x} \rightarrow X_{x}$ denote the base change $\mathscr{X} \times{ }_{S} x \rightarrow X \times{ }_{S} x$.

Proposition 4.5. Let $L$ be a line bundle on $\mathscr{X}$ such that for every field valued point $x: \operatorname{Spec}(k) \rightarrow S$, the sheaf $\pi_{x *}\left(\left.L\right|_{\mathscr{x}_{x}}\right)$ is locally free of rank 1 and $\pi_{x}^{*} \pi_{x *}\left(\left.L\right|_{\mathscr{P}_{x}}\right) \rightarrow$ $\left.L\right|_{\mathscr{X}_{x}}$ is an isomorphism. If $\mathscr{X} \rightarrow X$ is flat, then the sheaf $\pi_{*} L$ is locally free of rank 1 on $X$ and $\pi^{*} \pi_{*} L \rightarrow L$ is an isomorphism.

Proof. One immediately reduces to the case when $X=\operatorname{Spec}(R), Y=\operatorname{Spec}(B)$ is a finite flat $R$ scheme, and $\Gamma$ is a finite group acting on $Y$ over $X$ such that $\mathscr{X}=[Y / \Gamma]$ (see for example [Olsson 2006, 2.12]). Let $M$ denote the $B$-module corresponding to $L$, so that $M$ comes equipped with an action of $\Gamma$ over the action on $B$. We can even assume that $R$ is a local ring and that $M$ is a free $R$-module (forgetting the $B$-module structure). We are then trying to compute the kernel of the map

$$
M \rightarrow \prod_{\gamma \in \Gamma} M, \quad m \mapsto(\cdots, \gamma(m)-m, \cdots)_{\gamma \in \Gamma}
$$

We can also assume that $S=\operatorname{Spec}(\Lambda)$ is affine.

Lemma 4.6. Let $R$ be a reduced local $\Lambda$-algebra and let $A \in M_{n \times m}(R)$ be a matrix (which we view as a map $R^{n} \rightarrow R^{m}$ ) with the property that for every $x \in \operatorname{Spec}(\Lambda)$, the matrix $A(x) \in M_{n \times m}\left(R \otimes_{\Lambda} k(x)\right)$ has kernel a free $R \otimes_{\Lambda} k(x)$-space of rank 1. Then $\operatorname{Ker}(A)$ is a free rank 1 module over $R$ and for every $x \in \operatorname{Spec}(\Lambda)$, the natural map $\operatorname{Ker}(A) \otimes_{\Lambda} k(x) \rightarrow \operatorname{Ker}(A(x))$ is an isomorphism.

Proof by induction on $n$. If $n=1$, then the assertion is that $A$ is a matrix with $A(x)$ the zero matrix for all $x \in \operatorname{Spec}(\Lambda)$. Since $R$ is reduced this implies that $A$ is the zero matrix. 
For the inductive step, consider the system of $m$ equations

$$
\sum_{i} a_{i j} X_{i}=0
$$

that we are trying to solve in $R$. If $x \in \operatorname{Spec}(\Lambda)$ is the image of the closed point of $\operatorname{Spec}(R)$, then $A(x)$ is not zero since $n \geq 2$. Since $R$ is local, some $a_{i j}$ is invertible and so we can solve for the variable $X_{i}$. This gives a system of $m-1$ equations in $n-1$ variables, which again has the property that for every point $x \in \operatorname{Spec}(\Lambda)$, the image in $R \otimes k(x)$ has a unique line of solutions. By induction we obtain the result.

This completes the proof of Theorem 1.1(i).

\section{Proof of Theorem 1.1(ii)}

Proposition 5.1. For any scheme $S$ over $\mathbb{Z}[1 / 2]$, with $\pi: M_{1,1, S} \rightarrow \mathbb{A}_{j, S}^{1}$ the projection, and any coherent $O_{S}$-module $M$, the sheaf $R^{1} \pi_{*}\left(\mathcal{O}_{\mathcal{M}_{1,1, S}} \otimes_{\mathcal{O}_{S}} M\right)$ is zero.

Proof. Using the theorem of formal functions one is reduced to the case when $S$ is the spectrum of a field. Furthermore, if the characteristic is not 3, the result is immediate, as $M_{1,1, S}$ is tame in this case (see, for example, the proof of [Abramovich and Vistoli 2002, 2.3.4]), so it suffices to consider $S=\operatorname{Spec}(k)$ with $\operatorname{char}(k)=3$, and $M=k$. We may further assume that $k$ is algebraically closed.

The coherent sheaf $R^{1} \pi_{*}\left(\mathcal{O}_{\mu_{1,1, k}}\right)$ restricts to the zero sheaf on $\mathbb{A}_{k}^{1}-\{0\}$, since over this open subset of $\mathbb{A}_{k}^{1}$ the stack $\mathcal{M}_{1,1, k}$ is tame (the automorphism groups are $\{ \pm 1\})$. Let $\bar{x} \rightarrow M_{1,1, k}$ be a geometric point mapping to 0 in $\mathbb{A}_{k}^{1}$, and let $A$ denote the completion of $\mathcal{O}_{M_{1,1, l}, \bar{x}}$ along the maximal ideal. Let $\Gamma_{\bar{x}}$ denote the stabilizer group scheme of $\bar{x}$, so that $\Gamma_{\bar{x}}$ acts on $A$. The ring of invariants $B:=A^{\Gamma_{\bar{x}}}$ is equal to the completion of $\mathbb{A}_{k}^{1}$ at the origin. Let $F$ denote the finite type $B$-module obtained by pulling back $R^{1} \pi_{*}\left(\mathcal{O}_{\mu_{1,1, k}}\right)$ to $\operatorname{Spec}(B)$. Then $F$ is equal to the cohomology group $H^{1}\left(\Gamma_{\bar{x}}, A\right)$. We show that this group is zero. Since $F$ is supported on the closed point of $\operatorname{Spec}(B)$, there exists an integer $n$ such that $j^{n} F=0$ (where $j \in B$ is the uniformizer defined by the standard coordinate on $A^{1}$ ). To prove the proposition it therefore suffices to show that $F$ is $j$-torsion-free.

For this we use an explicit description of $A$ and $\Gamma_{\bar{x}}$ given by the Legendre family. Let

$$
V=\operatorname{Spec}(k[\lambda][1 / \lambda(\lambda-1)]),
$$

and let $E_{V} \rightarrow V$ be the elliptic curve

$$
E_{V}: Y^{2} Z=X(X-Z)(X-\lambda Z) .
$$

If $\mu$ denotes $\lambda+1$, then the $j$-invariant of $E_{V}$ is equal to $\mu^{6} /\left(\mu^{4}-1\right)$ (Recall that $\operatorname{char}(k)=3$.) The map $V \rightarrow M_{1,1, k}$ defined by $E_{V}$ is étale (see for example [Katz 
and Mazur 1985, 2.2.8]), so this defines an isomorphism $A \simeq k \llbracket \mu \rrbracket$. The group $\Gamma_{\bar{x}}$ sits in an exact sequence

$$
1 \rightarrow\{ \pm 1\} \rightarrow \Gamma_{\bar{x}} \rightarrow S_{3} \rightarrow 1
$$

and the action of $\Gamma_{\bar{x}}$ on $A \simeq k \llbracket \mu \rrbracket$ factors through the action of $S_{3}$ on $k \llbracket \mu \rrbracket$ given by the two automorphisms

$$
\alpha: \mu \mapsto-\mu
$$

and

$$
\beta: \mu \mapsto \mu /(1-\mu)=\mu\left(1+\mu+\mu^{2}+\ldots\right) .
$$

Also note that the Leray spectral sequence

$$
E_{2}^{p q}=H^{p}\left(S_{3}, H^{q}(\{ \pm 1\}, A)\right) \Longrightarrow H^{p+q}\left(\Gamma_{\bar{x}}, A\right)
$$

and the fact that $H^{q}(\{ \pm 1\}, A)=0$ for $q>0$ (since 2 is invertible in $k$ ) imply that $H^{1}\left(\Gamma_{\bar{x}}, A\right)=H^{1}\left(S_{3}, A\right)$.

An element in $H^{1}\left(S_{3}, A\right)$ can be represented by a set map $\xi: S_{3} \rightarrow k \llbracket \mu \rrbracket$ (written $\sigma \mapsto \xi_{\sigma}$ ) such that for $\sigma, \tau \in S_{3}$ we have (recall the action is a right action)

$$
\xi_{\sigma \tau}=\xi_{\sigma}^{\tau}+\xi_{\tau} .
$$

The class of $\xi$ is trivial if there exists an element $g \in k \llbracket \mu \rrbracket$ such that $\xi_{\sigma}=g^{\sigma}-g$ for all $\sigma \in S_{e}$. Note that (5-7) implies that it suffices to check the equalities $\xi_{\sigma}=g^{\sigma}-g$ for a set of generators $\sigma \in S_{3}$.

If $\xi$ represents a class in $H^{1}\left(S_{3}, A\right)$ annihilated by $j$, there exists an element $g \in k \llbracket \mu \rrbracket$ such that

$$
\frac{\mu^{6}}{\mu^{4}-1} \xi_{\sigma}=g^{\sigma}-g
$$

for all $\sigma \in S_{3}$. To prove that $H^{1}\left(S_{3}, A\right)$ is $j$-torsion-free, it therefore suffices to show that for such a $\xi$, we can choose $g$ to have $\mu$-adic valuation $\geq 6$ (since $A$ is $j$-torsion-free).

For this, note that we can assume without loss of generality that $g$ has no constant term, and then write

$$
g=a_{1} \mu+a_{2} \mu^{2}+a_{3} \mu^{3}+a_{4} \mu^{4}+a_{5} \mu^{5}+g_{\geq 6},
$$

where $g_{\geq 6}$ has $\mu$-adic valuation $\geq 6$. We have

$$
\frac{\mu^{6}}{\mu^{4}-1} \xi_{\alpha}=2 a_{1} \mu+2 a_{3} \mu^{3}+2 a_{5} \mu^{5}+\left(g_{\geq 6}^{\alpha}-g_{\geq 6}\right),
$$

which implies that $a_{1}=a_{3}=a_{5}=0$. Then

$$
\left.\frac{\mu^{6}}{\mu^{4}-1} \xi_{\beta}=2 a_{2} \mu^{3}+\text { (higher order terms }\right) \text {, }
$$


which gives $a_{2}=0$. Finally, using this, we see that

$$
\frac{\mu^{6}}{\mu^{4}-1} \xi_{\beta}=a_{4} \mu^{5}+(\text { higher order terms }),
$$

which implies that $a_{4}=0$ as desired. This completes the proof of Proposition 5.1.

5.2. Now let us prove Theorem 1.1 for a connected $\mathbb{Z}[1 / 2]$-scheme $S$. We need to show that if $L$ is a line bundle on $\mathcal{M}_{1,1, S}$ such that for any field-valued point $s \in S$ the fiber $L_{s}$ on $M_{1,1, s}$ descends to $\mathbb{A}_{j, s}^{1}$, then $L$ descends to $\mathbb{A}_{j, S}^{1}$. By a standard limit argument it suffices to consider the case when $S$ is noetherian and even affine, say $S=\operatorname{Spec}(\Lambda)$. Let $J \subset \Lambda$ denote the nilradical. By the reduced case already treated in Section 4, it suffices to show inductively that if the result holds for $\Lambda / J^{r}$ then it also holds for $\Lambda / J^{r+1}$. In other words, let $L_{0}$ denote a line bundle on $A_{j, \Lambda / J^{r}}^{1}$ and $\widetilde{L}$ a lifting of $\pi^{*} L_{0}$ to $M_{1,1, \Lambda / J^{r+1}}$. Then we want to show that $\widetilde{L}$ is pulled back from a lifting of $L_{0}$ to $\mathbb{A}_{j, \Lambda / J^{r+1}}^{1}$. By standard deformation theory this is equivalent to showing that the map

$$
0=H^{1}\left(\mathbb{A}_{\Lambda}^{1}, J^{r} / J^{r+1}\right) \rightarrow H^{1}\left(M_{1,1, \Lambda}, J^{r} / J^{r+1}\right)
$$

is an isomorphism; or, equivalently, that $H^{1}\left(M_{1,1, \Lambda}, J^{r} / J^{r+1}\right)$ is zero. Since $\mathbb{A}_{j, \Lambda}^{1}$ is affine, the group $H^{1}\left(M_{1,1, \Lambda}, J^{r} / J^{r+1}\right)$ is zero if and only if the sheaf $R^{1} \pi_{*}\left(J^{r} / J^{r+1} \otimes \mathcal{O}_{\mu_{1,1, \Lambda}}\right)$ is zero on $\mathbb{A}_{j, \Lambda}^{1}$, which follows from Proposition 5.1. This completes the proof of Theorem 1.1.

\section{Computations in characteristic 2}

Proposition 6.1. Let $k$ be a field of characteristic 2 , and let $\pi: \bar{M}_{1,1, k} \rightarrow \mathbb{P}_{k}^{1}$ be the morphism defined by the $j$-invariant. Then $R^{1} \pi_{*} \widehat{O}_{\bar{M}_{1,1, k}}$ is a line bundle on $\mathbb{P}_{k}^{1}$ of negative degree.

Proof. We may without loss of generality assume that $k$ is algebraically closed.

Let $\cup_{\infty} \subset \bar{M}_{1,1, k}$ denote the open substack $\bar{M}_{1,1, k} \times_{\mathbb{P}_{j}^{1}} \mathbb{A}_{1 / j}^{1}$ (the complement of $j=0)$, and let $\boldsymbol{U}_{0}=M_{1,1, k} \subset \bar{M}_{1,1, k}$ denote the complement of $j=\infty$. Let $U_{\infty}, U_{0} \subset \mathbb{P}_{j}^{1}$ be the coarse moduli spaces (the standard open cover of $\mathbb{P}_{j}^{1}$ ).

The stack $U_{\infty}$ is a $\mathbb{Z} /(2)$-gerbe over $U_{\infty}$. Now in general, if $f: \varphi \rightarrow X$ is a $\mathbb{Z} /(2)$-gerbe in characteristic 2 , the sheaf $R^{1} f_{*} \mathrm{O}_{\varphi}$ is locally free of rank 1 and in fact canonically trivialized. This can be seen as follows. Étale locally on $X$, we have $\mathscr{G}=X \times B(\mathbb{Z} /(2))$. Computing in this local situation, one sees that $R^{1} f_{*}(\mathbb{Z} /(2))$ is a locally constant sheaf of groups étale locally isomorphic to $\mathbb{Z} /(2)$, and the natural map $R^{1} f_{*}(\mathbb{Z} /(2)) \otimes_{\mathbb{Z} /(2)} O_{X} \rightarrow R^{1} f_{*} \mathrm{O}_{\varphi}$ (which exists since we are in characteristic 2 ) is an isomorphism. Since a group of order 2 admits no nontrivial automorphisms, there is a canonical isomorphism $\mathbb{Z} /(2) \simeq R^{1} f_{*}(\mathbb{Z} /(2))$ which induces a canonical 
trivialization of $R^{1} f_{*} \mathrm{O}_{g}$. In the case of $\varphi=X \times B(\mathbb{Z} /(2))$ and $X=\operatorname{Spec}(A)$ we have

$$
H^{1}\left(\varphi, O_{\varphi}\right) \simeq \operatorname{Hom}_{\mathrm{Gp}}(\mathbb{Z} /(2), A),
$$

and the trivialization is given by the homomorphism sending $1 \in \mathbb{Z} /(2)$ to $1 \in A$.

Lemma 6.2. The sheaf $R^{1} \pi_{*} \mathcal{O}_{\bar{M}_{1,1, k}}$ is locally free of rank 1 on $\mathbb{P}_{j}^{1}$.

Proof. By finiteness of coherent cohomology for stacks, the sheaf is in any case coherent. Since $\mathbb{P}_{j}^{1}$ is a smooth curve it therefore suffices to show that it is torsionfree. Furthermore, the only issue is at the point $j=0$. Since the formation of cohomology commutes with flat base change, it suffices to show that

$$
H^{1}\left(\bar{M}_{1,1, k} \times_{\mathbb{P}_{j}^{1}} \operatorname{Spec}(k \llbracket j \rrbracket), \mathcal{O}_{\bar{M}_{1,1, k} \times_{\mathbb{P}} j} \operatorname{Spec}(k \llbracket j \rrbracket)\right.
$$

is $j$-torsion-free.

For this we use the so-called Hesse presentation of $M_{1,1, k}$. Let

$$
V=\operatorname{Spec}\left(k[\mu, \omega]\left[1 /\left(\mu^{3}-1\right)\right] /\left(\omega^{2}+\omega+1\right)\right),
$$

and let $E_{V} \rightarrow V$ be the elliptic curve given by the equation

$$
X^{3}+Y^{3}+Z^{3}=\mu X Y Z .
$$

This elliptic curve has a basis for its three-torsion group given by the points $[1: 0$ : $-1]$ and $[-1: \omega: 0]$. In fact, this is the universal elliptic curve with full level three structure. The $j$-invariant of $E_{V}$ is $\mu^{12} /\left(\mu^{3}-1\right)^{3}$ (since we are in characteristic 2). In particular, the fiber over $j=0$ is the curve $X^{3}+Y^{3}+Z^{3}=0$.

Changing the choice of basis for the 3-torsion subgroup defines an action of $G L_{2}\left(\mathbb{F}_{3}\right)$ on $V$ such that $M_{1,1, k} \simeq\left[V / G L_{2}\left(\mathbb{F}_{3}\right)\right]$. A calculation shows that this action is described as follows:

(1) $(\mu, \omega) *\left(\begin{array}{rr}1 & 0 \\ -1 & 1\end{array}\right)=(\omega \mu, \omega)$.

(2) $(\mu, \omega) *\left(\begin{array}{rr}0 & -1 \\ 1 & 0\end{array}\right)=(\mu /(\mu-1), \omega)$.

(3) $(\mu, \omega) *\left(\begin{array}{rr}1 & 0 \\ 0 & -1\end{array}\right)=\left(\mu, \omega^{2}\right)$.

Putting this together one finds that

$$
\bar{M}_{1,1, k} \times_{\mathbb{P}_{j}^{1}} \operatorname{Spec}(k \llbracket j \rrbracket) \simeq\left[\operatorname{Spec}(k \llbracket \mu \rrbracket) / \operatorname{SL}_{2}\left(\mathbb{F}_{3}\right)\right],
$$

where $\alpha=\left(\begin{array}{rr}1 & 0 \\ -1 & 1\end{array}\right)$ acts by $\mu \mapsto \zeta \mu$ (for some fixed primitive cube root of unity $\zeta$ ) and $\beta=\left(\begin{array}{cc}0 & -1 \\ 1 & 0\end{array}\right)$ acts by $\mu \mapsto \mu /(\mu-1)$.

As in the proof of Proposition 5.1, an element of (6-1) is given by a set map $\xi: \mathrm{SL}_{2}\left(\mathbb{F}_{3}\right) \rightarrow k \llbracket \mu \rrbracket\left(\right.$ written $\left.\sigma \mapsto \xi_{\sigma}\right)$ such that for any two elements $\sigma, \tau \in \mathrm{SL}_{2}\left(\mathbb{F}_{3}\right)$ we have

$$
\xi_{\sigma \tau}=\xi_{\sigma}^{\tau}+\xi_{\tau}
$$


and the class of $\xi$ is trivial if there exists an element $g \in k \llbracket \mu \rrbracket$ such that for every $\sigma$ we have $\xi_{\sigma}=g^{\sigma}-g$.

Now if (6-1) has $j$-torsion there exists a set map $\xi$ as above and an element $g \in k \llbracket \mu \rrbracket$ such that for all $\sigma$ we have

$$
\frac{\mu^{12}}{\mu^{3}-1} \xi_{\sigma}=g^{\sigma}-g
$$

To prove that (6-1) is torsion-free, it suffices to show that we can choose $g$ to be divisible by $\mu^{12}$. For since $k \llbracket \mu \rrbracket$ is an integral domain we then have

$$
\xi_{\sigma}=\left(\frac{\mu^{3}-1}{\mu^{12}} g\right)^{\sigma}-\left(\frac{\mu^{3}-1}{\mu^{12}} g\right) .
$$

We can without loss of generality assume that $g$ has no constant term. Write

$$
g=a_{1} \mu+a_{2} \mu^{2}+\cdots+a_{11} \mu^{11}+g_{\geq 12} .
$$

Then $g^{\alpha}-g$ has $\mu$-adic valuation $\geq 12$ (recall that $\alpha=\left(\begin{array}{rr}1 & 0 \\ -1 & 1\end{array}\right)$ ). Looking at the coefficients $a_{i}$, this implies that all but $a_{3}, a_{6}$, and $a_{9}$ are zero, so

$$
g=a_{3} \mu^{3}+a_{6} \mu^{6}+a_{9} \mu^{9}+g_{\geq 12} .
$$

Similarly, $g^{\beta}-g$ has $\mu$-adic valuation $\geq 12$. Looking at the coefficient of $\mu^{4}$ in $g^{\beta}-g$ one sees that $a_{3}=0$. Then looking at the coefficent of $\mu^{8}$ one sees that $a_{6}=0$, and finally looking at the coefficient of $\mu^{10}$ one sees that $a_{9}=0$.

Let $M$ denote the cohomology group (6-1) (a $k \llbracket j \rrbracket-$ module) and let $M_{\eta}$ denote $M \otimes_{k \llbracket j \rrbracket} k \llbracket j \rrbracket[1 / j]$. Let $e_{\infty} \in M_{\eta}$ denote the basis element defined by the canonical trivialization of $R^{1} \pi_{*} \mathcal{O}_{\bar{M}_{1,1, k}}$ over $\boldsymbol{U}_{\infty}$. The lattice $M \subset M_{\eta}$ defines a valuation $v$ on $M_{\eta}$ and it suffices to show that $v\left(e_{\infty}\right)<0$. Equivalently, we have to show that for any element $m \in M$, if we write $m=h e_{\infty}$ in $M_{\eta}$ then the $j$-adic valuation of $h$ is positive.

For this we again use the presentation (6-4). An element $m \in M$ is then represented by a map $\xi: \mathrm{SL}_{2}\left(\mathbb{F}_{3}\right) \rightarrow k \llbracket \mu \rrbracket$. The corresponding element in $M_{\eta}$ can be described in terms of the basis $e_{\infty}$ as follows. First of all, the element $\xi_{\beta^{2}} \in k \llbracket \mu \rrbracket$ is $\mathrm{SL}_{2}\left(\mathbb{F}_{3}\right)$-invariant, since for any other element $\sigma$ we have

$$
\xi_{\beta^{2}}^{\sigma}+\xi_{\sigma}=\xi_{\beta^{2} \sigma}=\xi_{\sigma \beta^{2}}=\xi_{\sigma}^{\beta^{2}}+\xi_{\beta^{2}}
$$

and $\beta^{2}$ acts trivially on $k \llbracket \mu \rrbracket$. Therefore, $\xi_{\beta^{2}}$ is actually an element in $k \llbracket j \rrbracket$. The image of $\xi$ in $M_{\eta} \simeq \operatorname{Hom}(\mathbb{Z} /(2), k \llbracket j \rrbracket[1 / j])$ is then equal to the homomorphism

$$
\mathbb{Z} /(2) \rightarrow k \llbracket j \rrbracket[1 / j], \quad 1 \mapsto \xi_{\beta^{2}} .
$$


The class $e_{\infty}$ corresponds to the homomorphism sending 1 to 1 , so we have to show that the $j$-adic valuation of $\xi_{\beta^{2}}$ is positive. For this, let $f=\xi_{\beta}$. Then

$$
\xi_{\beta^{2}}=f^{\beta}+f=f\left(\mu\left(1+\mu+\mu^{2}+\cdots\right)\right)+f(\mu) .
$$

Since we are in characteristic 2 , it follows that the $\mu$-adic valuation of $\xi_{\beta^{2}}$ is at least 2 , and therefore the $j$-adic valuation of $\xi_{\beta^{2}}$ is also positive.

Corollary 6.3. For any field $k$, we have $H^{1}\left(\bar{M}_{1,1, k}, \mathcal{O}_{\bar{M}_{1,1, k}}\right)=0$.

Proof. We have $R^{1} \pi_{*} O_{\bar{M}_{1,1, k}}=0$ when $\operatorname{char}(k) \neq 2$ (When $\operatorname{char}(k)=3$, this follows from Proposition 5.1.) It follows that

$$
H^{0}\left(\mathbb{P}_{k}^{1}, R^{1} \pi_{*} \mathcal{O}_{\bar{M}_{1,1, k}}\right)=0
$$

in all characteristics. From the Leray spectral sequence we obtain

$$
H^{1}\left(\bar{M}_{1,1, k}, \mathbb{O}_{\bar{M}_{1,1, k}}\right)=H^{1}\left(\mathbb{P}_{k}^{1}, \mathbb{O}_{\mathbb{P}_{k}^{1}}\right)=0 .
$$

This concludes the proof.

Remark 6.4. Note that if $\operatorname{char}(k)=2$, then the restriction of $R^{1} \pi_{*} \mathcal{O}_{\mu_{1,1, k}}$ to $\mathbb{A}_{k}^{1} \subset \mathbb{P}_{k}^{1}$ is nonzero. From the Leray spectral sequence it follows that the map

$$
0=H^{1}\left(\mathbb{A}_{k}^{1}, \mathrm{O}_{\mathbb{A}_{k}^{1}}\right) \rightarrow H^{1}\left(M_{1,1, k}, \mathcal{O}_{M_{1,1, k}}\right)
$$

is not an isomorphism. Since the group $H^{1}\left(M_{1,1, k}, \mathcal{O}_{M_{1,1, k}}\right)$ classifies deformations of the structure sheaf to $M_{1,1, k[\epsilon] /\left(\epsilon^{2}\right)}$, this implies that there are line bundles on $M_{1,1, k[\epsilon] /\left(\epsilon^{2}\right)}$ which are in the kernel of (4-1) but are nontrivial. This implies that Theorem 1.1 fails for $S=\operatorname{Spec}\left(k[\epsilon] /\left(\epsilon^{2}\right)\right)$. More generally, Theorem 1.1 fails for any nonreduced affine scheme over $\mathbb{F}_{2}$.

\section{Proof of Theorem 1.3}

7.1. In order to prove Theorem 1.3 it is easiest to prove a stronger statement that implies it. Let $\mathscr{P} i c\left(\bar{M}_{1,1, S}\right)$ denote the Picard stack over $S$ which to any $S$-scheme $T$ associates the group of line bundles on $\bar{M}_{1,1, T}$. By [Aoki 2006, 5.1], the stack $\mathscr{P} \operatorname{ic}\left(\bar{M}_{1,1, S}\right)$ is an algebraic stack (an Artin stack) over $S$. There is a morphism of stacks

$$
\mathbb{Z} \times B \mathbb{G}_{m, S} \rightarrow \mathscr{P i c}\left(\bar{M}_{1,1, S}\right)
$$

sending a pair $(n, L)$ consisting of $n \in \mathbb{Z}$ and $L$ a line bundle on $S$ to $\lambda^{n} \otimes_{\mathcal{O}_{S}} L$ on $\bar{M}_{1,1, S}$. The following theorem implies Theorem 1.3 by evaluation of both sides of (7-1) on $S$ and passing to isomorphism classes.

Theorem 7.2. The morphism (7-1) is an isomorphism. 
Proof. Note first that if $n$ and $n^{\prime}$ are integers and $L$ and $L^{\prime}$ are line bundles on $S$, then $\lambda^{n} \otimes L$ and $\lambda^{n^{\prime}} \otimes L^{\prime}$ on $\bar{M}_{1,1, S}$ are isomorphic if and only if $n=n^{\prime}$ and $L \simeq L^{\prime}$. Indeed, if these two sheaves are isomorphic, then this implies that $\lambda^{n-n^{\prime}}$ descends to $\mathbb{P}_{j}^{1}$. By the case of a field, this implies that $n=n^{\prime}$. In this case we recover $L$ and $L^{\prime}$ from their pullbacks to $\bar{M}_{1,1, S}$ by pushing back down to $S$. Therefore, the functor (7-1) is fully faithful. It therefore suffices to show that for any cartesian diagram

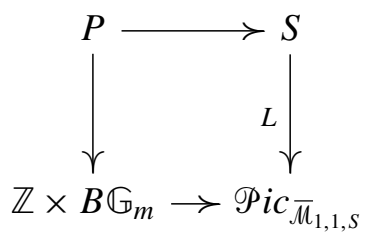

the morphism of algebraic spaces $P \rightarrow S$ is an isomorphism. For this it suffices to consider the case when $S$ is artinian local. Furthermore, we know the result in the case when $S$ is the spectrum of a field by Section 3. Since a line bundle on the spectrum of an artinian local ring is trivial, what we therefore need to show is that if $S$ is an artinian local ring, then any line bundle on $\bar{M}_{1,1, S}$ is isomorphic to $\lambda^{n}$ for some $n$. Proceeding by induction on the length of $S$, it further suffices to consider the following. Let $S=\operatorname{Spec}(A), k$ the residue field of $A$, and let $J \subset A$ be a square-zero ideal annihilated by the maximal ideal of $A$, and set $A_{0}=A / J$. Then any deformation of $\lambda^{n}$ over $\bar{M}_{1,1, A_{0}}$ to $\bar{M}_{1,1, A}$ is isomorphic to $\lambda^{n}$. Using the exponential sequence

$$
0 \rightarrow J \otimes \mathcal{O}_{\bar{M}_{1,1, k}} \rightarrow \mathrm{O}_{\bar{M}_{1,1, A}}^{*} \rightarrow \mathrm{O}_{\bar{M}_{1,1, A}}^{*} \rightarrow 0
$$

one sees that this amounts exactly to $H^{1}\left(\bar{M}_{1,1, k}, \mathcal{O}_{\bar{M}_{1,1, k}}\right)=0$.

\section{References}

[Abramovich and Vistoli 2002] D. Abramovich and A. Vistoli, "Compactifying the space of stable maps", J. Amer. Math. Soc. 15:1 (2002), 27-75. MR 2002i:14030 Zbl 0991.14007

[Aoki 2006] M. Aoki, "Hom stacks", Manuscripta Math. 119:1 (2006), 37-56. MR 2007a:14003 Zbl 1094.14001

[Deligne 1975] P. Deligne, "Courbes elliptiques: formulaire d'après J. Tate”, pp. 53-73 in Modular functions of one variable (Antwerp, 1972), vol. IV, edited by B. J. Birch and W. Kuyk, Lecture Notes in Math. 476, Springer, Berlin, 1975. MR 52 \#8135

[Fulton 1998] W. Fulton, Intersection theory, vol. 2, 2nd ed., Springer, Berlin, 1998. MR 99d:14003 Zbl 0885.14002

[Ischebeck 1979] F. Ischebeck, "Über die Abbildung Pic $A \rightarrow \operatorname{Pic} A_{f}$ ", Math. Ann. 243:3 (1979), 237-245. MR 80k:14015 Zbl 0418.14008

[Katz and Mazur 1985] N. M. Katz and B. Mazur, Arithmetic moduli of elliptic curves, vol. 108, Princeton University Press, 1985. MR 86i:11024 Zbl 0576.14026 
[Keel and Mori 1997] S. Keel and S. Mori, "Quotients by groupoids", Ann. of Math. (2) 145:1 (1997), 193-213. MR 97m:14014 Zbl 0881.14018

[Mumford 1965] D. Mumford, "Picard groups of moduli problems", pp. 33-81 in Arithmetical Algebraic Geometry (West Lafayette, IN), edited by O. F. G. Schilling, Harper \& Row, New York, 1965. MR 34 \#1327 Zbl 0187.42801

[Olsson 2005] M. C. Olsson, "On proper coverings of Artin stacks", Adv. Math. 198:1 (2005), 93106. MR 2006h:14003 Zbl 1084.14004

[Olsson 2006] M. C. Olsson, "Hom-stacks and restriction of scalars", Duke Math. J. 134:1 (2006), 139-164. MR 2007f:14002 Zbl 1114.14002

[Silverman 2009] J. H. Silverman, The arithmetic of elliptic curves, 2nd ed., Graduate Texts in Mathematics 106, Springer, Dordrecht, 2009. MR 2514094 Zbl 05549721

Communicated by Ravi Vakil

Received 2009-05-15 Revised 2009-09-30 Accepted 2009-11-30

wfulton@umich.edu Department of Mathematics, University of Michigan, Ann Arbor, MI 48109-1043, United States

molsson@math.berkeley.edu Department of Mathematics, Univesity of California, Berkeley, CA 94720-3840, United States 